Annales Mathematicae Silesianae 32 (2018), 65-78

DOI: 10.1515/amsil-2017-0012

\title{
INFINITE TOWERS OF GALOIS DEFECT EXTENSIONS OF KAPLANSKY FIELDS
}

\author{
ANNA BLASZCZOK
}

\begin{abstract}
We give conditions for Kaplansky fields to admit infinite towers of Galois defect extensions of prime degree. As proofs of the presented facts are constructive, this provides examples of constructions of infinite towers of Galois defect extensions of prime degree. We also give a constructive proof of the fact that a henselian Kaplansky field cannot be defectless-by-finite.
\end{abstract}

\section{Introduction}

In this paper, we denote by $(K, v)$ a field $K$ equipped with a valuation $v$. Its value group will be denoted by $v K$ and its residue field by $K v$. If $L$ is a field extension of $K$, then by $(L \mid K, v)$ we denote a valued field extension, where $v$ is a valuation of $L$ and $K$ is equipped with the restriction of $v$ to $K$.

Assume that $(L \mid K, v)$ is a finite extension of valued fields and the valuation $v$ of $K$ admits a unique extension to the field $L$. Then

$$
[L: K]=p^{n} \cdot(v L: v K)[L v: K v]
$$

where $n$ is a nonnegative integer and, by the Lemma of Ostrowski, $p$ is the characteristic exponent of $K v$, that is, $p=\operatorname{char} K v$ if it is positive and $p=1$ otherwise. The factor $d(L \mid K, v)=p^{n}$ is called the defect of the extension

Received: 01.04.2017. Accepted: 08.11.2017. Published online: 31.01.2018.

(2010) Mathematics Subject Classification: 12J10, 12J25.

Key words and phrases: defect extensions, Kaplansky fields, Artin-Schreier defect extensions, Kummer defect extensions, defectless-by-finite fields. 
$(L \mid K, v)$. If $d(L \mid K, v)>1$ then $L \mid K$ is called a defect extension. If $d(L \mid K, v)=1$ then we call $L \mid K$ a defectless extension.

A valued field $(K, v)$ is called henselian if $v$ extends in a unique way to a valuation of the algebraic closure $\widetilde{K}$ of $K$, or equivalently, to every algebraic extension of $K$. If in addition every finite extension of $(K, v)$ is defectless, then the valued field is called defectless.

A better understanding of defect extensions and their structure turned out to be important for deep open problems of the model theory of valued fields as well as the theory of valued rational function fields (see, e.g., [11, [13] and [14]).

Since the existence of defect extensions shows a "bad behaviour" of the valuation, we are interested in the question whether the problem of defect extensions appears only in finite extensions, after which the defect vanishes. In other words, we are interested if there are finite extensions $(L \mid K, v)$ of henselian fields such that $(K, v)$ is not defectless, but $(L, v)$ is a defectless field. Such a field $(K, v)$ is called defectless-by-finite.

A valued field extension $(L \mid K, v)$ is called immediate if the corresponding value group and residue field extensions are trivial, i.e.,

$$
(v L: v K)=[L v: K v]=1 .
$$

Note that finite immediate extensions of henselian fields are defect extensions. If a valued field admits no nontrivial immediate extensions, then it is called maximal. Hence, a maximal immediate extension of a valued field is a maximal field.

In [8] Krull proved that every valued field admits a maximal immediate extension. The structure and uniqueness of maximal immediate extensions of valued fields are important topics, in particular in connection with the applications to the model theory of valued fields.

A valued field $(K, v)$ of residue characteristic $p$ is called a Kaplansky field if it satisfies the following conditions:

(K1) if $p>0$ then the value group $v K$ is $p$-divisible,

(K2) the residue field $K v$ is perfect,

(K3) the residue field $K v$ admits no finite separable extension of degree divisible by $p$.

Note that every valued field of residue characteristic 0 satisfies the above conditions, hence is a Kaplansky field. It was shown by Kaplansky that if a valued field $(K, v)$ satisfies (K1)-(K3), then it admits a unique (up to valuation preserving isomorphism over $K$ ) maximal immediate extension. For the proof see Theorem 5 of [6]. See also Theorem 1 of [16], which shows the equivalence of conditions (K1) - (K3) with the original "hypothesis A" assumed by Kaplansky. 
He gave also an example of a valued field admitting nonisomorphic maximal immediate extensions (cf. [6, Section 5]).

The uniqueness of maximal immediate extensions of Kaplansky fields was a key tool in the study of the model theory of Kaplansky fields. Moreover, defectless Kaplansky fields are examples of so-called tame fields (for the definition see Section 2.2. The algebraic properties of tame fields made it possible to prove strong model-theoretical properties of the elementary class of tame fields and have been applied to questions related with algebraic geometry ([9],[14,,15]). Theorem 2.4 of [4] shows that if $(K, v)$ is a henselian Kaplansky field admitting a finite extension which is a defectless field, then $(K, v)$ is itself a defectless field. This shows that a Kaplansky field cannot be defectless-byfinite. Hence, if a Kaplansky field admits a defect extension, then it admits an infinite tower of defect extensions. However, the proof in [4] does not show us possible constructions of such towers.

One of the important tasks is to better understand the structure of defect extensions. Towers of Galois defect extensions of prime degree play a representative role for defect extensions. This follows from the fact that every finite separable extension $(L \mid K, v)$ of henselian fields, lifted up to a certain tame extension of $(K, v)$, becomes a tower of Galois extensions of degree equal to the characteristic exponent of $K v$. Furthermore, the defect of the lifted extension remains unchanged (for the details see Section 2.2. Therefore, we are interested in examples of constructions of towers of Galois defect extensions of prime degree.

In connection with problems related to resolution of singularities, such as local uniformization, we are interested in the structure and construction of defect extensions of rational function fields. The case of valued rational function fields of positive characteristic $p$ and $p$-divisible value group was studied in [1. That paper presents examples of constructions of infinite towers of Artin-Schreier defect extensions of such fields. Examples of Artin-Schreier defect extensions in the case of rational function fields with non- $p$-divisible value group are presented in [2]. Note that for fields of characteristic $p>0$, Artin-Schreier defect extensions are precisely the Galois defect extensions of degree $p$ (cf. Theorem 2.4).

In the present paper we give conditions for Kaplansky fields to admit infinite towers of Galois defect extensions of prime degree. More precisely, we show that if a Kaplansky field of positive characteristic $p$ admits a Galois defect extension then it admits an infinite tower of such extensions (cf. Theorem 3.2). We prove a counterpart of the theorem for the class of Kaplansky fields of characteristic 0 with positive residue characteristic $p \neq 2$ which contain a primitive $p$-th root of unity. We also give conditions for a valued field of characteristic 0 and residue characteristic 2 to admit a infinite tower of defect extensions of degree 2 (cf. Theorem 3.6. Corollary 3.7 and Corollary 3.8). As all of the proofs are constructive, this provides examples for the further 
study of defect extensions, in particular in the case of function fields which are Kaplansky fields (which for instance can happen when they are immediate extensions of Kaplansky base fields).

The facts mentioned above allow us to give a constructive alternative proof of the fact that Kaplansky fields cannot be defectless-by-finite (cf. Corollary 3.10 .

\section{Preliminaries}

\subsection{Galois extensions of prime degree}

Take a field $K$. The algebraic closure of $K$ will be denoted by $\tilde{K}$. Take a prime number $p$. If $p \neq \operatorname{char} K$, then denote by $\varepsilon_{p}$ a primitive $p$-th root of unity in $\tilde{K}$. We recall the form of Galois extensions of $K$ of degree $p$. Note that since $p$ is a prime number, Galois extensions of $K$ of degree $p$ are precisely cyclic extensions of $K$ of degree $p$.

We start with the case of char $K \neq p$. A field extension $E \mid K$ is called irreducible radical if it is generated by a root of an irreducible polynomial $X^{n}-a \in K[X]$.

THEOREM 2.1. If $K$ is a field of characteristic distinct from $p$ containing a primitive $p$-th root of unity, then a field extension $E \mid K$ of degree $p$ is cyclic if and only if it is irreducible radical.

For the proof see Lemma 1.1, Chapter 8 of [7].

Thus if char $K \neq p$ and $\varepsilon_{p} \in K$, then cyclic extensions of degree $p$ are precisely the extensions generated by roots of irreducible polynomials $X^{p}-a \in$ $K[X]$. The next lemmas give conditions for such binomials to be irreducible (cf. Theorem 1.6 and Lemma 1.5, Chapter 8 of [7]).

Lemma 2.2. Let $L$ be an arbitrary field. Take an element $a \in L$ and assume that the prime $p$ is odd. Then for any natural number $n$, the polynomial $X^{p^{n}}-a$ is irreducible over $L$ if and only if $a \notin L^{p}$.

Lemma 2.3. Assume that $F$ is a field of characteristic distinct from 2. Take an element $a \in F$ and a natural number $n \geq 2$. Then the polynomial $X^{2^{n}}-a$ is irreducible over $F$ if and only if $a \notin F^{2}$ and $a \notin-4 F^{4}$.

We are left with the case of char $K=p$. An Artin-Schreier extension of $K$ is an extension of degree $p$ generated by a root $\vartheta$ of a polynomial $X^{p}-X-a$ 
with $a \in K$. In this case, $\vartheta$ is called an Artin-Schreier generator of the extension. For the proof of the following theorem see Theorem 2.7, Chapter 7 of [7].

Theorem 2.4. Assume that $K$ is a field of characteristic $p$.

(i) A field extension $E \mid K$ of degree $p$ is cyclic if and only if it is an ArtinSchreier extension.

(ii) If $a \in K$, then the polynomial $X^{p}-X-a$ is either irreducible or splits into distinct linear factors over $K$.

LEMma 2.5. Take a field $L$ of positive characteristic $p$ and an element $a \in L$. Assume that the polynomial $f=X^{p}-X-a$ is irreducible over L. If $\vartheta$ is a root of $f$, then the polynomial $X^{p}-X-a \vartheta^{p-1}$ is irreducible over $L(\vartheta)$.

For the proof see Lemma 1.10, Chapter 8 of [7].

\subsection{Tame and defect extensions}

Recall that a valued field $(K, v)$ is called henselian, if $v$ extends in a unique way to a valuation of the algebraic closure of $K$. For every valued field $(K, v)$ there is a minimal henselian field extension of $(K, v)$, in the sense that it admits a valuation preserving embedding over $K$ in every henselian extension of $(K, v)$. Such an extension is unique up to valuation preserving isomorphism over $K$ (cf. Theorem 17.11 of [5]). We call it the henselization of $(K, v)$ and denote by $(K, v)^{h}$ or, if $v$ is fixed, by $K^{h}$. The extension $K^{h} \mid K$ is always immediate and separable-algebraic (cf. [5], Theorem 17.19). Furthermore, if $L$ is any algebraic extension of $K$ and $v$ is a fixed extension from $K$ to the algebraic closure of $K$, then

$$
L^{h}=L \cdot K^{h}
$$

Lemma 2.6. Take a valued field $(K, v)$ and a finite extension $L$ of $K$. Then the extension of $v$ to $L$ is unique if and only if $L \mid K$ is linearly disjoint from some (equivalently, every) henselization of $(K, v)$.

For the proof see Lemma 2.1 of [4].

Assume that $(K, v)$ is a Kaplansky field of residue characteristic $p>0$. Note that this is equivalent to the conjunction of the following two conditions: (K1) the value group $v K$ is $p$-divisible,

(K2') the residue field $K v$ admits no finite extension of degree divisible by $p$. 
Take a finite extension $L$ of the field and assume that $v$ extends in a unique way from $K$ to $L$. Let $[L: K]=p^{n} m$, where $m$ is coprime with $p$ and $n \geq 0$. Then

$$
p^{n} m=[L: K]=d(L \mid K, v)(v L: v K)[L v: K v] .
$$

Note that the conditions (K1) and (K2') imply that the ramification index $(v L: v K)$ as well as the inertia degree $[L v: K v]$ are coprime with $p$ and consequently $d(L \mid K, v)=p^{n}$. We thus obtain the following fact.

Lemma 2.7. Assume that $(K, v)$ is a Kaplansky field of a positive residue characteristic $p$. Take a finite extension $(L \mid K, v)$ such that the valuation $v$ extends in a unique way from $K$ to $L$.

a) If the degree of $L \mid K$ is divisible by $p$, then the extension has a nontrivial defect. More precisely, if $L \mid K$ is of degree $p^{n} m$, where $n>0$ and $p$ does not divide $m$, then $d(L \mid K, v)=p^{n}$.

b) If $[L: K]=p^{n}$ for some natural number $n$, then $d(L \mid K, v)=[L: K]$ and the extension is immediate.

Assume that $(K, v)$ is a henselian field. A finite extension $(E, v)$ of $(K, v)$ is called tame if it satisfies the following conditions:

(T1) the ramification index $(v E: v K)$ is prime to the characteristic exponent of $K v$,

(T2) the residue field extension $E v \mid K v$ is separable,

(T3) $(E \mid K, v)$ is a defectless extension.

An algebraic extension $(L, v)$ of $(K, v)$ is called tame if every finite subextension $(E \mid K, v)$ of $(L \mid K, v)$ is tame. Directly from the above definition it follows that every tame extension of valued fields is separable-algebraic. A henselian field is called tame if every algebraic extension of the field is tame. Note that if $(L \mid K, v)$ is an algebraic extension of Kaplansky fields, then conditions (T1) and (T2) are always satisfied. Hence an algebraic extension of a henselian Kaplansky field is tame if and only if every finite subextension of the extension is defectless. Moreover, a henselian Kaplansky field is tame if and only if it is defectless.

The next two facts are crucial for the study of the structure of defect extensions. Ramification theory enables us to prove the following well known property.

Lemma 2.8. Take a henselian field $(K, v)$ of positive residue characteristic $p$ and a finite normal extension $L$ of $K$. Then there is a finite tame subextension $N \mid K$ of $L \mid K$ such that $L \mid N$ is a tower of normal extensions of degree $p$. 
Proof. Denote by $E$ the relative separable algebraic closure of $K$ in $L$ and by $N$ the fixed field of the subgroup

$$
G^{r}:=\left\{\sigma \in \operatorname{Gal}(E \mid K) \mid v(\sigma a-a)>v a \text { for all } a \in \mathcal{O}_{K^{\text {sep }}} \backslash\{0\}\right\}
$$

of $\operatorname{Gal}(E N \mid K)$. Then Theorem 5.8 of [10] yields that $E \mid N$ is a tower of normal extensions of degree $p$. As $L \mid E$ is a purely inseparable extension, it is also a tower of normal extensions of degree $p$. Moreover, Theorems 5.7 and 5.8 of [10] show that $N \mid K$ is a tame extension.

Lemma 2.9. Take a henselian field $(K, v)$ and a finite extension $L \mid K$. Then for any tame extension $N$ of $K$,

$$
d(L \mid K, v)=d(L \cdot N \mid N, v) .
$$

For the proof see Proposition 2.8 of [12].

Assume that $(K, v)$ is a henselian field and char $K v=p>0$. Take a finite separable defect extension $(L \mid K, v)$ of henselian fields. Without loss of generality we can assume that $L \mid K$ is normal. Otherwise we replace $L$ by the normal hull of $L$ over $K$. Then, by Lemma 2.8 there is a finite tame subextension $N \mid K$ of $L \mid K$ such that $L \mid N$ is a tower of Galois extensions of degree $p$. Moreover, by Lemma 2.9 we have $d(L \mid K, v)=d(L \mid N, v)$. Thus if $L \mid K$ has nontrivial defect, then the tower $L \mid N$ contains a Galois defect extension of prime degree. If in addition $(K, v)$ is a Kaplansky field, then Lemma 2.7 yields that $d(L \mid N, v)=[L: N]$ and thus $L \mid N$ is a nontrivial tower of Galois defect extensions of degree $p$.

\section{Towers of Galois defect extensions of prime degree}

We consider now the case of Kaplansky fields of positive residue characteristic. We show that the existence of at least one defect extension of prime degree of a Kaplansky field $(K, v)$ of positive characteristic implies the existence of an infinite tower of such extensions. In the case of Kaplansky fields of characteristic 0 and positive residue characteristic we prove the theorem under additional assumptions.

We treat first the case of valued fields of positive characteristic. We start with general remarks about the existence of infinite towers of Galois extensions of degree $p$ for any field of positive characteristic $p$. As for such a field an extension of degree $p$ is Galois if and only if it is an Artin-Schreier extension 
(cf. Theorem 2.4), we consider the existence of infinite towers of Artin-Schreier extensions.

Lemma 3.1. Assume that $K$ is a field of positive characteristic. If it admits an Artin-Schreier extension, then it admits already an infinite tower of such extensions.

Proof. Assume that $K_{0}:=K$ admits an Artin-Schreier extension $K_{1}$ of degree $p=\operatorname{char} K$. Then $K_{1}=K_{0}\left(\vartheta_{1}\right)$, where $\vartheta_{1}$ is a root of an irreducible polynomial $f_{1}=X^{p}-X-a_{1} \in K[X]$. Consider the following construction. For every $n \geq 1$ :

$$
\left\{\begin{array}{l}
\text { if } K_{n}=K_{n-1}\left(\vartheta_{n}\right) \text { with } \vartheta_{n}^{p}-\vartheta_{n}=a_{n} \in K_{n-1}, \\
\text { take } K_{n+1}=K_{n}\left(\vartheta_{n+1}\right) \text { with } \vartheta_{n+1} \text { a root of } \\
f_{n+1}:=X^{p}-X-a_{n} \vartheta_{n}^{p-1} \text { and set } a_{n+1}=a_{n} \vartheta_{n}^{p-1} .
\end{array}\right.
$$

Take a natural number $n$ and suppose that $K_{n} \mid K_{n-1}$ is an Artin-Schreier extension, that is, the polynomial $f_{n}=X^{p}-X-a_{n}$ is irreducible over $K_{n-1}$. Then Lemma 2.5 yields that the polynomial $f_{n+1}$ is irreducible over $K_{n}$ and $K_{n+1} \mid K_{n}$ is an Artin-Schreier extension.

By induction on $n$ we obtain an infinite tower of Artin-Schreier extensions $K_{n} \mid K_{n-1}, n \in \mathbb{N}$.

We now apply the above result to the valued field extensions of Kaplansky fields.

TheOREM 3.2. Take a Kaplansky field $(K, v)$ of positive characteristic. Assume that $K$ admits an Artin-Schreier extension $E \mid K$ such that $v$ extends in a unique way to a valuation of $E$. Then $(E \mid K, v)$ has nontrivial defect and $(K, v)$ admits an infinite tower of Artin-Schreier defect extensions containing $E$.

Proof. Set $p:=$ char $K$. Since $K_{1}:=E$ is an Artin-Schreier extension of $K$, it is of the form $K\left(\vartheta_{1}\right)$, where $\vartheta_{1}$ is a root of a polynomial $f_{1}=X^{p}-X-a_{1} \in K[X]$. From Lemma 3.1 we deduce that $K_{0}:=K$ admits an infinite tower of Artin-Schreier extensions $K_{n} \mid K_{n-1}$, which can be obtained by construction (3.1).

By our assumption, the valuation $v$ admits a unique extension from $K_{0}$ to $K_{1}$. Take $n \in \mathbb{N}$ and assume that we have already shown that $v$ admits a unique extension from $K_{n-1}$ to $K_{n}$. From Lemma 2.6 it follows that $K_{n} \mid K_{n-1}$ is linearly disjoint from $K_{n-1}^{h} \mid K_{n-1}$. Hence $\left[K_{n-1}^{h}\left(\vartheta_{n}\right): K_{n-1}^{h}\right]=p$ and thus the polynomial $f_{n}$ is irreducible over $K_{n-1}^{h}$. By Lemma 2.5 the polynomial 
$f_{n+1}$ is irreducible over $K_{n-1}^{h}\left(\vartheta_{n}\right)=K_{n}^{h}$. Hence $\left[K_{n}^{h}\left(\vartheta_{n+1}\right): K_{n}^{h}\right]=p$ and $K_{n+1} \mid K_{n}$ is linearly disjoint from $K_{n}^{h} \mid K_{n}$. Applying again Lemma 2.6 we deduce that $v$ admits a unique extension from $K_{n}$ to $K_{n+1}$. By induction on $n$, this holds for every extension in the tower.

Take a natural number $n$. Since $(K, v)$ is a Kaplansky field, the same holds for $\left(K_{n-1}, v\right)$. Hence, Lemma 2.7 yields that $\left(K_{n} \mid K_{n-1}, v\right)$ is an immediate extension and $d\left(K_{n} \mid K_{n-1}, v\right)=p$. As $E=K_{1}$, we have that also $(E \mid K, v)$ is a defect extension.

Constructions of infinite towers of Artin-Schreier defect extensions were studied also in [1]. For the proof of the following fact see Theorem 1.4 of that paper.

THEOREM 3.3. Take a valued field $(L, v)$ of positive characteristic $p$ which satisfies (K1) and (K2). If there is a purely inseparable extension of $(L, v)$ which does not lie in the completion of the field, then $L$ admits an infinite tower of Artin-Schreier defect extensions.

Note that if $(K, v)$ is a Kaplansky field of positive characteristic $p$, then $v K$ is $p$-divisible and $K v$ is perfect. Hence, Theorem 3.3 gives another condition for Kaplansky field of positive characteristic to admit an infinite tower of Artin-Schreier defect extension. Since the proof of the theorem is constructive, it gives an alternative constructions of such a tower. Moreover, in Theorem 3.2 we require the existence of an Artin-Schrier (hence separable) defect extension, whereas in Theorem 3.3 we require the existence of a certain purely inseparable defect extension to obtain an infinite tower of Artin-Schreier defect extensions.

We consider now the case of valued fields of characteristic 0 and positive residue characteristic $p$. We will study separately the case of odd primes and $p=2$.

As in the case of valued fields of positive characteristic, we start with general remarks about the existence of infinite towers of Galois extensions of degree $p$ for the case of fields of characteristic zero. We will assume that the fields admit primitive $p$-th root of unity. Hence we will investigate when such fields admit infinite towers of irreducible radical extensions of degree $\mathrm{p}$ (cf. Theorem 2.1).

The next two well known facts will be a basis of our constructions. We treat first the case of an odd prime $p$.

Lemma 3.4. Assume that $K$ is a field of characteristic 0. Take an odd prime $p$ and assume that $K$ contains a primitive $p$-th root of unity. If $K$ admits a Galois extension of degree $p$, then it admits an infinite tower of such extensions. 
Proof. Set $K_{0}:=K$ and assume that $K_{1}$ is a Galois extension of $K_{0}$ of degree $p$. Since $\varepsilon_{p} \in K$, we have that $K_{1}=K_{0}\left(\eta_{1}\right)$, where $\eta_{1}$ is a root of an irreducible polynomial $f_{1}:=X^{p}-a \in K_{0}[X]$. By Lemma 2.2 we have $a \notin K_{0}^{p}$. Applying again Lemma 2.2 we obtain that for every natural number $n$ the polynomial

$$
f_{n}:=X^{p^{n}}-a
$$

is irreducible over $K_{0}$. Denote by $K_{n}, n>1$ the extension of $K_{0}$ generated by a root $\eta_{n}$ of the polynomial $f_{n}$. Then $\left[K_{n}: K_{o}\right]=p^{n}, n \in \mathbb{N}$. Hence each of the extensions $K_{n} \mid K_{n-1}$ is of degree $p$. Assume additionally that we have chosen the roots $\eta_{n}$ in a way that $\eta_{n+1}^{p}=\eta_{n}, n \in \mathbb{N}$. Then for every natural number $n \geq 2$, the extension $K_{n} \mid K_{n-1}$ is generated by a root $\eta_{n}$ of the polynomial $X^{p}-\eta_{n-1} \in K_{n-1}[X]$. As $\left[K_{n}: K_{n-1}\right]=p$, the extension $K_{n} \mid K_{n-1}$ is irreducible radical, hence Galois.

It remains to consider the case of char $K=0$ and $p=2$. Note that in this case every extension of degree 2 is Galois.

Lemma 3.5. Take a field $K$ of characteristic 0 and assume that $K$ admits an extension $K_{1}$ of degree 2 distinct from $K(i)$, where $i$ is a square root of -1 . Then the field admits an infinite tower of extensions of degree 2.

Proof. Set $K_{0}=K$. Then the extension $K_{1} \mid K_{0}$ is generated by a root $\eta_{1}$ of an irreducible polynomial $f_{1}=X^{2}-a \in K[X]$. Hence, $a$ is not a square in $K$. Suppose that $\eta_{1}^{2}=a \in-4 K^{4}$. Then $\eta_{1}= \pm 2 i b^{2}$ for some $b \in K$. Since $\eta_{1} \notin K$, we obtain that $i \in K\left(\eta_{1}\right) \backslash K$. Thus $K_{1}=K\left(\eta_{1}\right)=K(i)$, a contradiction. Therefore $a \notin-4 K^{4}$ and Lemma 2.3 yields that for every $n \geq 2$ the polynomial

$$
f_{n}:=X^{2^{n}}-a
$$

is irreducible over $K_{0}$. As in the proof of Lemma 3.4 we construct an infinite tower of extensions of degree 2.

Note that assertion of the above lemma may not hold for $K_{1}=K(i)$. Indeed, if $K$ is a real closed field, then $K(i) \mid K$ is an extension of degree 2, but $K(i)$ is algebraically closed.

We are now able to prove the counterpart of Theorem 3.2 in the case of Kaplansky fields of characteristic 0 and positive residue characteristic.

THEOREM 3.6. Take a Kaplansky field $(K, v)$ of characteristic 0 and with char $K v=p>0$. Assume that one of the following cases holds: 
1) $p \neq 2$, the field $K$ contains a primitive $p$-th root $\varepsilon_{p}$ of unity and admits a Galois extension $E$ of degree $p$ such that $v$ extends in a unique way to a valuation of $E$,

2) $p=2$, the field $K$ admits an extension $E$ of degree 2 not contained in the henselization of $K(i)$.

Then $(E \mid K, v)$ has defect equal to the degree of the extension and $(K, v)$ admits an infinite tower $F$ of Galois extensions of degree and defect $p$ such that $E \subseteq F$.

Proof. Fix an extension of the valuation $v$ to $\tilde{K}$ and call it again $v$. Set $K_{0}=K$.

In each of the cases $K_{0}$ admits a Galois extension $E$ of degree $p$ and the valuation $v$ extends in a unique way from $K_{0}$ to $E$. As $\varepsilon_{p} \in K_{0}$, the extension is generated by a root $\eta_{1}$ of a polynomial $f_{1}=X^{p}-a \in K_{0}[X]$. Moreover, if $p=2$, then by assumption $E \neq K(i)$. From Lemmas 3.4 and 3.5 it follows that $K_{0}$ admits an infinite tower of Galois extensions $K_{n} \mid K_{n-1}$ of degree $p$. Furthermore, we can choose $K_{1}=E$ and $K_{n}=K_{0}\left(\eta_{n}\right)$, where $\eta_{n}$ is a root of a polynomial (3.2) or (3.3), depending on char $K v$, and $\eta_{n}^{p}=\eta_{n-1}$, for every $n \geq 2$.

Since by assumption the valuation $v$ extends uniquely to $K_{1}=K_{0}\left(\eta_{1}\right)$, by Lemma 2.6 we have that $K_{1}$ is linearly disjoint from $K_{0}^{h}$ over $K_{0}$, and we obtain that $\left[K_{0}^{h}\left(\eta_{1}\right): K_{0}^{h}\right]=p$ and $f_{1}$ is irreducible over $K_{0}^{h}$. Moreover, if $p=2$, then by assumption $\eta_{1} \notin K(i)^{h}=K^{h}(i)$. Hence, $\eta_{1} \notin \pm 2 i\left(K^{h}\right)^{2}$ and thus $a \notin-4\left(K^{h}\right)^{4}$. By Lemmas 2.2 and 2.3 , this yields that also the polynomials $f_{n}, n \geq 2$, are irreducible over $K_{0}^{h}$. Hence, $\left[K_{0}^{h}\left(\eta_{n}\right): K_{0}^{h}\right]=p^{n}$ and the extensions $K_{n} \mid K_{0}$ and $K_{0}^{h} \mid K_{0}$ are linearly disjoint. It follows that $v$ has a unique extension from $K_{n-1}$ to $K_{n}$ for every $n$.

As in the proof of Theorem 3.2 we deduce that each of the extensions $K_{n} \mid K_{n-1}$ is immediate and thus has defect equal to the degree of the extension. This yields that in each of the cases $(K, v)$ admits an extension $F$ which is an infinite tower of Galois extensions of degree and defect $p$. Moreover, by our construction $E \subseteq F$.

Corollary 3.7. Take a Kaplansky field $(K, v)$ of characteristic 0 and residue characteristic 2. Assume that one of the following cases holds:

2 ) the field $K$ contains a square root $i$ of -1 and admits an extension $E$ of degree 2 such that $v$ extends in a unique way to a valuation of $E$,

$3)$ the field $K$ admits an extension $E$ which is a tower of two extensions of degree 2 and $v$ extends in a unique way to a valuation of $E(i)$.

Then $(E \mid K, v)$ has defect equal to the degree of the extension and $(K, v)$ admits an infinite tower $F$ of defect extensions of degree 2 such that $E \subseteq F$. 
Proof. Fix an extension of $v$ to $\tilde{K}$ and call it again $v$. The assumptions yield that $E(i) \mid K(i)$ is either an extension of degree 2 or a tower $K(i) \subseteq L \subseteq$ $E(i)$ of two such extensions. In the first case set $K^{\prime}=K(i)$; in the latter one take $K^{\prime}=L$. Furthermore, in both cases set $E^{\prime}=E(i)$.

Since $v$ extends in a unique way from $K$ to $E^{\prime}$, it extends also in a unique way from $K^{\prime}$ to $E^{\prime}$. Thus $E^{\prime} \nsubseteq K^{\prime h}=K^{\prime}(i)^{h}$. Hence, Theorem 3.6 yields that $\left(K^{\prime}, v\right)$ admits an extension $F$ which is an infinite tower of extensions of degree and defect 2. By our construction $E \subseteq F$. Furthermore, since $(K, v)$ is a Kaplansky field and $v$ admits a unique extension from $K$ to $K^{\prime}$, we deduce that $\left(K^{\prime} \mid K, v\right)$ is a defect extension of degree 2 or a tower of two such extensions. Hence $F \mid K$ is an infinite tower of extensions of degree and defect 2.

Note that if $i \notin K$ and $K(i) \mid E$ is an extension of degree 2 such that $v$ extends in a unique way from $K$ to $E$ but not to $E(i)$, we may have that $E \subseteq$ $K(i)^{h}$, that is $E^{h}=K(i)^{h}=K^{h}(i)$. Then the assumptions of Lemma2.3 may not be satisfied for $F=K^{h}$. Thus the construction presented in Theorem 3.6 may not give an infinite tower of defect extensions. However, the next corollary shows that we can construct such a tower over the henselization of $K$.

Corollary 3.8. Assume that $(K, v)$ is a henselian Kaplansky field of characteristic 0 and residue characteristic 2 . If it admits a defect extension $L$ of degree 2 , then it admits an infinite tower of such extensions containing $L$.

Proof. Note that since $(K, v)$ is henselian, $v$ extends in a unique way to any algebraic extension of $K$.

Assume that $K$ contains a square root $i$ of -1 . Then, applying part 2) of Corollary 3.7 to $E=L$ we obtain that $(K, v)$ admits an infinite tower of defect extensions of degree 2 containing $L$. Assume that $L$ admits an extension $E$ of degree 2. Then $E \mid K$ is a tower of two extensions of degree 2, thus the assertion of the corollary follows from part 3 ) of Corollary 3.7 .

It remains to consider the case when $i \notin K$ and $L$ admits no extensions of degree 2. Then $L=K(i)$, as otherwise $L(i) \mid L$ would be an extension of degree 2. Take $a, b \in K$. Since $K(i)$ admits no extensions of degree $2, a+b i$ is a square of some element $\eta=c+d i$ with $c, d \in K$. Then $a^{2}+b^{2}=\left(c^{2}+d^{2}\right)^{2} \in K^{2}$. Hence, every sum of squares in $K$ is a square in $K$. Moreover $-1 \notin\left(K^{\times}\right)^{2}=$ $\sum\left(K^{\times}\right)^{2}$, as $i \notin K$. Therefore, $K$ is a formally real field. Since $(K, v)$ is henselian, Lemma 2.3 of [4] yields that char $K v=0$, a contradiction.

We use the above facts to show that if a henselian Kaplansky field $(K, v)$ admits any defect extension $(E \mid K, v)$, then it admits an infinite tower of defect extensions. As the proof is based on the above theorems, we obtain in particular a possible construction of an extension of $(K, v)$ of arbitrarily high defect. 
COROLlary 3.9. Assume that $(K, v)$ is a henselian Kaplansky field of positive residue characteristic $p$ which admits a finite normal defect extension $(L \mid K, v)$. Then there is a finite tame subextension $N \mid K$ of $L \mid K$ which admits an infinite tower of normal defect extensions of degree $p$ containing $L$.

Proof. Since $L \mid K$ and $K\left(\varepsilon_{p}\right) \mid K$ are normal extensions, $L . K\left(\varepsilon_{p}\right)$ is also a normal extension of $K$. Moreover, $K\left(\varepsilon_{p}\right) \mid K$ is of degree dividing $p-1$ and thus is a tame extension. By the multiplicativity of the defect, we obtain that $d\left(L\left(\varepsilon_{p}\right) \mid K, v\right)=d(L \mid K, v)$. Hence we can replace $L$ by $L\left(\varepsilon_{p}\right)$.

By Lemma 2.8 there is a finite tame subextension $N \mid K$ of $L \mid K$ such that $L \mid N$ is a tower of normal extensions of degree $p$. Note that $\varepsilon_{p} \in N$. Moreover, $(N, v)$ is a henselian Kaplansky field, as already $(K, v)$ is. Hence, by Lemma 2.7 each of the extensions in the tower $L \mid N$ is immediate and thus is a defect extension. Together with Lemma 2.9 this yields that

$$
[L: N]=d(L \mid N, v)=d(L \mid K, v) .
$$

Therefore, the extension $L \mid N$ is nontrivial.

Assume first that $(L \mid N, v)$ is not separable. Then in particular char $N=p$ and the field $N$ is not perfect. Consequently, $L$ is not perfect, as $L \mid N$ is a finite extension. Take $a \in L$ such that $a^{1 / p} \notin L$. Set $F=L\left(a^{1 / p^{i}} \mid i \in \mathbb{N}\right)$. Since $v L$ is $p$-divisible and $L v$ is perfect, $F \mid L$ is an infinite tower of purely inseparable defect extensions of degree $p$. Thus $F \mid N$ is an infinite tower of normal defect extension of degree $p$.

Assume now that $(L \mid N, v)$ is a separable extension. Then we obtain a tower $N=: L_{0} \subseteq L_{1} \subseteq \ldots \subseteq L_{m}:=L$, where $L_{j} \mid L_{j-1}$ is a Galois defect extension of degree $p$ for every $j \in\{1, \ldots, m\}$. If char $K=p$, then $\left(L_{m} \mid L_{m-1}, v\right)$ satisfies the assumptions of Theorem 3.2. Assume that char $K=0$. Then the extension $\left(L_{m} \mid L_{m-1}, v\right)$ satisfies the assumptions of Theorem 3.6 or Corollary 3.8 . depending on the residue characteristic. Hence, in each of the cases we deduce that $L_{m-1}$ admits an infinite tower of Galois defect extensions of degree $p$ containing $L_{m}=L$. Thus already $N=L_{0}$ admits such a tower.

Take $(E \mid K, v)$ to be any finite defect extension of a henselian Kaplansky field of positive residue characteristic $p$. If $L$ is the normal hull of $E \mid K$, then $L \mid K$ satisfies the assumptions of the above corollary. Consequently, there is a finite tame extension $N \mid K$ such that the field $(N, v)$ admits an extension $(F, v)$ which is an infinite tower of normal defect extensions of degree $p$. Furthermore, $F$ can be chosen such that $E \subseteq F$. This shows in particular that $(E, v)$ is not defectless. We thus have proved the following fact.

Corollary 3.10. A henselian Kaplansky field cannot be defectless-byfinite. 


\section{References}

[1] Blaszczok A., Infinite towers of Artin-Schreier defect extensions of rational function fields, in: A. Campillo, F.-V. Kuhlmann, B. Teissier (Eds.), Valuation theory in interaction, EMS Series of Congress Reports, European Mathematical Society (EMS), Zürich, 2014, pp. 16-54.

[2] Blaszczok A., Distances of elements in valued field extensions, submitted.

[3] Blaszczok, A., Kuhlmann F.-V., Algebraic independence of elements in immediate extensions of valued fields, J. Algebra 425 (2015), 179-214.

[4] Blaszczok A., Kuhlmann F.-V., On maximal immediate extensions of valued fields, Math. Nachr. 290 (2017), 7-18.

[5] Endler O., Valuation theory, Springer-Verlag, Berlin, 1972.

[6] Kaplansky I., Maximal fields with valuations, Duke Math. J. 9 (1942), 303-321.

[7] Karpilovsky G., Topics in field theory, North-Holland Mathematics Studies 155, North-Holland Publishing Co., Amsterdam, 1989.

[8] Krull W., Allgemeine Bewertungstheorie, J. Reine Angew. Math. 167 (1932), 160-196.

[9] Kuhlmann F.-V., Henselian function fields and tame fields, preprint (extended version of Ph.D. thesis), Heidelberg, 1990.

[10] Kuhlmann F.-V., Valuation theoretic and model theoretic aspects of local uniformization, in: H. Hauser, J. Lipman, F. Oort, A. Quirós (Eds.), Resolution of singularities. A research textbook in tribute to Oscar Zariski, Progress in Mathematics 181, Birkhäuser Verlag, Basel, 2000, pp. 381-456.

[11] Kuhlmann F.-V., Value groups, residue fields, and bad places of rational function fields, Trans. Amer. Math. Soc. 356 (2004), 4559-4600.

[12] Kuhlmann F.-V., A classification of Artin-Schreier defect extensions and characterizations of defectless fields, Illinois J. Math. 54 (2010), 397-448.

[13] Kuhlmann F.-V., The defect, in: M. Fontana, S.-E. Kabbaj, B. Olberding, I. Swanson (Eds.), Commutative algebra. Noetherian and non-Noetherian perspectives, SpringerVerlag, New York, 2011, pp. 277-318.

[14] Kuhlmann F.-V., The algebra and model theory of tame valued fields, J. Reine Angew. Math. 719 (2016), 1-43.

[15] Kuhlmann F.-V., Elimination of ramification II: henselian rationality of valued function fields, in preparation.

[16] Whaples G., Galois cohomology of additive polynomial and $n$-th power mappings of fields, Duke Math. J. 24 (1957), 143-150.

Institute of Mathematics

University of Silesia

BANKOWA 14

40-007 Katowice

POLAND

e-mail: anna.blaszczok@us.edu.pl 